\title{
Investigation of Strouhal number of ice-accreted bridge cables at moderate flow turbulence
}

\author{
Piotr Górski ${ }^{1, *}$, Marcin Tatara $^{1}$, Stanislav Pospíšil ${ }^{2}$ and Sergej Kuznetsov ${ }^{2}$ \\ ${ }^{1}$ Opole University of Technology, Faculty of Civil Engineering and Architecture, Department \\ of Roads and Bridges, Katowicka 48, Opole 45-061, Poland \\ ${ }^{2}$ Academy of Sciences of the Czech Republic, Institute of Theoretical and Applied Mechanics, Czech \\ Republic
}

\begin{abstract}
This paper is concerned with the investigation of the Strouhal number $(S t)$ for a stationary iced cable model of cable-supported bridges using wind tunnel tests with respect to three principal angles of wind attack. The investigations were carried out in the Climatic Wind Tunnel Laboratory of the Czech Academy of Sciences in Telč. The $S t$ values were determined at the flow turbulence intensity of the order of $12 \%$, within the range of the Reynolds number from $2.4 \cdot 10^{4}$ to $12.9 \cdot 10^{4}$, based on the dominant vortex shedding frequencies measured in the wake of the model. The obtained experimental $S t$ values of the iced cable of a cable-supported bridge will allow to determine the critical wind velocity as well as will be helpful for prediction of the cable response due to the vortex excitation phenomenon at moderate flow turbulence condition.
\end{abstract}

\section{Introduction}

It is well known that the vortex street is formed in the wake behind a body and the vortexshedding frequency is characterized by the Strouhal number $(S t)$. The knowledge of the $S t$ value is necessary for determination of the critical wind velocity at which the largest amplitude of body vibration due to vortex excitation are observed. It is also known that $S t$ strongly depends on the body shape, its motion, flow turbulence intensity and may in some cases depends on the Reynolds number $(R e)$. In the case of a body with the circular crosssection, e.g. a dry bridge cables, such dependences are well recognized [1]. It is noteworthy that previous wind tunnel studies, e.g. [2], show that the increase of the surface roughness of the cylinder as well as the increase of the turbulence intensity of free stream induce transition to the critical and transcritical regimes at a lower Re. Moreover, the greater flow turbulence may also produces the greater $S t$ values [3]. In the case of highly roughened cylinder the critical regime can be strongly reduced or may almost disappear.

In some weather conditions, the circular cross-section of a bridge cables may change due to ice accretion. In this case, the full-scale monitoring of slender bridge elements proved that ice accretion on the bridge cables has a significant influence on the flow field around the cables and their aerodynamics and can leads to a much larger amplitude of cable

\footnotetext{
*Corresponding author: p.gorski@po.opole.pl
} 
vibrations under wind action than without the ice [4]. It should be noticed that the literature concerning the icing influence on aerodynamics of cables of cable supported bridges is still limited, e.g. [5-8]. Therefore it is very advisable to conduct further studies to quantify the cable aerodynamic variation due to ice accretion. In the existing literature, the investigation results of $S t$ of iced bridge cables are presented in the paper [9] at low flow turbulence intensity with the value of $3 \%$. However, in most practical applications the flow field is usually turbulent with the turbulence intensity in the range between $10-20 \%$. It means that the mentioned experimental results of $S t$, received at low flow turbulence, cannot be applied in practice without any corrections.

This paper is concerned with the investigation of $S t$ for a stationary iced cable model of cable-supported bridges using wind tunnel tests with respect to three principal angles of wind attack at moderate flow turbulence. The investigations were carried out in the Climatic Wind Tunnel Laboratory of the Czech Academy of Sciences in Telč. One of the more frequent weather conditions in order to obtain the experimental icing of the inclined cable model was simulated. The ice shape of the cable was registered by a photogrammetry method. For the investigations of $S t$, a new iced cable model was made at a scale of 1:1.6 using a $3 \mathrm{D}$ printing procedure. The St values were determined at the flow turbulence intensity of the order of $12 \%$, within the range of the Reynolds number from $2.4 \cdot 10^{4}$ to $12.9 \cdot 10^{4}$, based on the dominant vortex-shedding frequencies measured in the wake of the model. The correctness of the $S t$ investigations was proved based on the $S t$ test of a circular smooth cylinder with the same experimental arrangement. Good agreement with the results reported in the existing literature was obtained. The obtained experimental $S t$ values of the iced cable of a cable-supported bridge will allow to determine the critical wind velocity as well as will be helpful for prediction of the cable response due to the vortex excitation phenomenon at moderate flow turbulence condition.

\section{The ice accretion process on the cable model}

The experimental icing process of a cable section model was conducted in a climatic section of the closed-return Climatic Wind Tunnel Laboratory of the Czech Academy of Sciences in Telč [10]. The $2.5 \mathrm{~m}$ long cable model as a pipe with a circular cross-section and $0.160 \mathrm{~m}$ diameter was inclined at an angle of $30^{\circ}$ in the vertical plane, at an angle of $60^{\circ}$ in the horizontal plane with respect to the wind velocity direction, and in this position was fixed to a special frame (see Fig. 1a). The model was made of polyvinylchloride (PVC), which surface is similar to the surface of a cable cover made of high-density polyethylene (HDPE). The icing process was performed at the average temperature about $-2{ }^{\circ} \mathrm{C}$, the mean free stream velocity $2.8 \mathrm{~m} / \mathrm{s}$, the rainy conditions using rain sprinklers of the diameter heads $2.8 \mathrm{~mm}$, and during a $40 \mathrm{~min}$ time period.

The final ice shape on the bottom side of the cable was obtained as the characteristic irregularly ice ribs with rounded edges and a relative surface roughness of $18 \%$ (Fig. 1b). On the upper part of the model the ice shape was similar to the circular shape with a surface roughness of $0.73 \%$. The cross-section of the cable with ice became strongly nonsymmetrical with the dimensions of $0.192 \mathrm{~m}$ height and $0.181 \mathrm{~m}$ width.

Immediately after the icing process the shape of the iced cable model was registered by a photogrammetry method. After a numerical image analysis a three-dimensional (3D) numerical model of the iced cable was received (Fig. 1c). For the St investigations the new iced cable model, shown in Fig. 1d, was made of polylactide plastic at a scale of 1:1.6 using a $3 \mathrm{D}$ printing procedure. The outer dimensions of the model cross-section were $0.120 \mathrm{~m}$ in height and $0.113 \mathrm{~m}$ in width while the model length was $0.43 \mathrm{~m}$. The descriptions of the wind tunnel, the icing process of the cable model in the climatic section, the final icing 
effect and preparation of the new iced cable model for the wind tunnel investigations are presented in the paper [9] in detail.
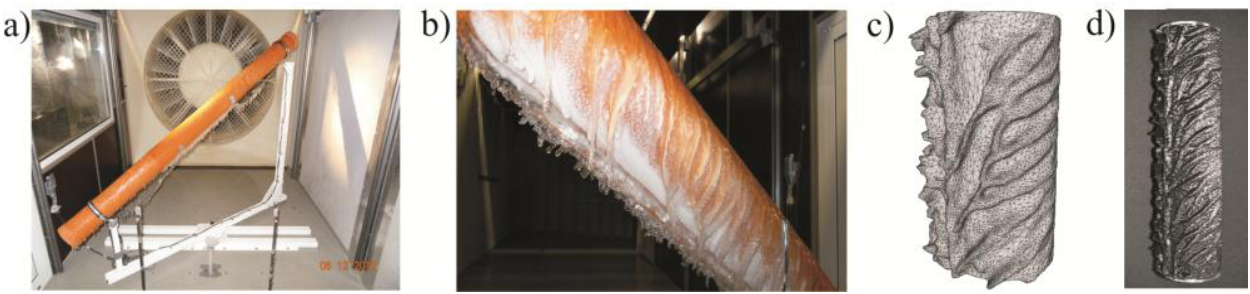

Fig. 1. (a) View of the cable model fixed to the special frame in the climatic section, (b) final icing effect of the cable model, (c) 3D numerical model of the ice shape of the cable model, and (d) new iced cable model for wind tunnel investigations made using a 3D printing method.

\section{Experimental investigations of Strouhal number}

The St investigations were carried out in the aerodynamic section of the wind tunnel. During the tests, the iced cable model was fixed horizontally in a special frame at a level of $69 \mathrm{~cm}$ above the floor of the aerodynamic section, crosswise to the airflow. Two sides of the frame were equipped with the plexi-glass end-plates to ensure two-dimensional flow around the model [11]. The height and the length of the plates were both $140.5 \mathrm{~cm}$, the width was $17 \mathrm{~cm}$ and their spacing was $47 \mathrm{~cm}$. The turbulent flow condition was modelled using a wooden grid with square bars placed $2.07 \mathrm{~m}$ upstream the model [12]. The width and the thickness of the grid bars was $5.5 \mathrm{~cm}$ and $4 \mathrm{~cm}$, respectively, and their spacing was $18 \mathrm{~cm}$. The experimental arrangement in the aerodynamic chamber is shown in Fig. 2.

a)

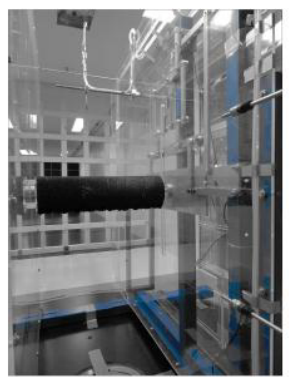

b)

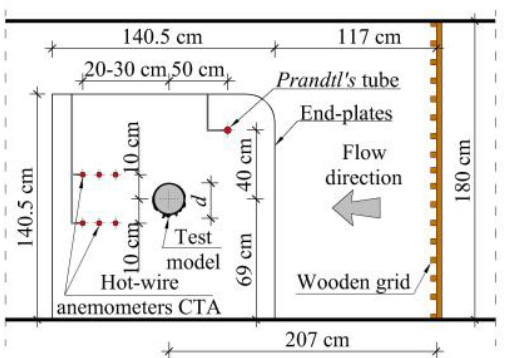

c)

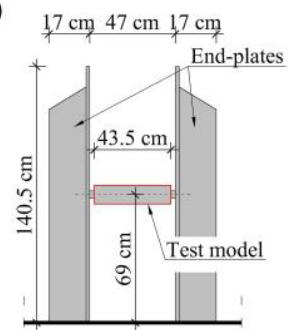

Fig. 2. (a) View of the model fixed to the special frame and the turbulence wooden grid, and sketch of the experimental arrangement (b) along and (c) across the aerodynamic section of the wind tunnel.

The St investigations were conducted under the assumption that the mean wind direction during the icing process is independent of the wind direction during the aerodynamic investigations. Thus, the $S t$ values were examined for three principal positions (configurations) of the ice cable model in relation to the flow direction which are presented in Fig. 3. In this figure the reference dimension $d$ perpendicular to the airflow direction of each model configuration are also shown.
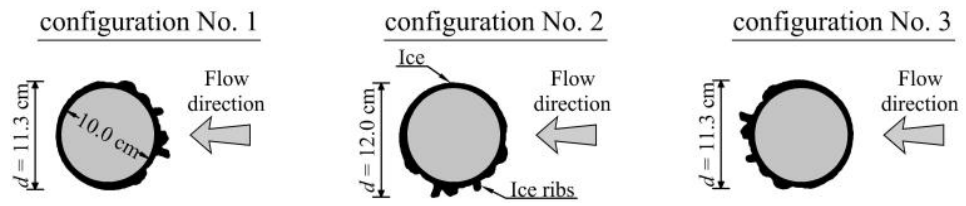

Fig. 3. Model configurations and their reference dimension $d$ perpendicular to the airflow direction considered in the $S t$ investigations. 
During the investigations the reference free stream velocity in front of the model $u$ was measured by the Prandtl's tube while the horizontal along-wind velocity component, at a distance of $1.7 \cdot d$ to $2.6 \cdot d(20$ to $30 \mathrm{~cm}$ ) behind the model, was measured by two hot-wire anemometers CTA (Constant Temperature Anemometry) over a $30 \mathrm{~s}$ interval. The sampling rate of data acquisition was set as $1000 \mathrm{~Hz}$. Two CTA sensors were fixed $10 \mathrm{~cm}$ above and below the longitudinal axis of the model (see Fig. 2b). The $S t$ values were determined based on the dominant vortex-shedding frequency $f_{s}$ extracted from the Power Spectral Density (PSD) of the airflow velocity measured in the wake of the model according to the formula

$$
S t=\frac{f_{s} \cdot d}{\bar{u}}
$$

where $f_{s}$ is the vortex-shedding frequency, $d$ is the reference dimension of the iced model perpendicular to the wind direction given in Fig. 3, and $\bar{u}$ is the mean free stream velocity in front of the model.

The $R e$ values were evaluated according to the formula

$$
\operatorname{Re}=\frac{\rho \cdot d \cdot \bar{u}}{\mu},
$$

where $\rho$ is the air density depends on the atmospheric pressure of the air and the air temperature, $\mu$ is the dynamic viscosity of the air depends on the air temperature.

The $\rho$ and $\mu$ values were calculated based on the actual air temperature and the atmospheric pressure of the air inside the aerodynamic section measured continuously during the tests. It was found that the air temperature in the aerodynamic chamber was changeable within the range from 25.1 to $31.7{ }^{\circ} \mathrm{C}$ whereas the atmospheric pressure was $958.2 \mathrm{hPa}$. The tests were carried out at ten mean free stream velocities $\bar{u}$ in the range from 3.5 up to $18.0 \mathrm{~m} / \mathrm{s}$. Consequently, the corresponding Re number regime in the interval $R e=2.4 \cdot 10^{4}$ to $12.9 \cdot 10^{4}$ was obtained.

The turbulence flow characterization in the test chamber was carried out using one CTA sensor. In this case, the measurements were performed in front of the model between the end-plates of the special frame revealing a longitudinal wind turbulence intensity upstream the center of the model. The turbulence intensity $T_{i}$ calculated as a ratio between the standard deviation of free stream velocity to mean value of the velocity was found about $12 \%$ and was classified as a moderately turbulent wind. The comparison of the turbulence spectra measured in front of the model between the end-plates of the special frame in a smooth flow, i.e. without the turbulence grid $\left(T_{i}=3 \%\right)$, and a moderate turbulent flow, i.e. with the turbulence grid, is depicted in Fig. 4. Due to the presence of the turbulence grid in the test chamber the free stream velocity decreased about $25 \%$ in relation to the same power of a ventilator which propels the airflow in the wind tunnel.

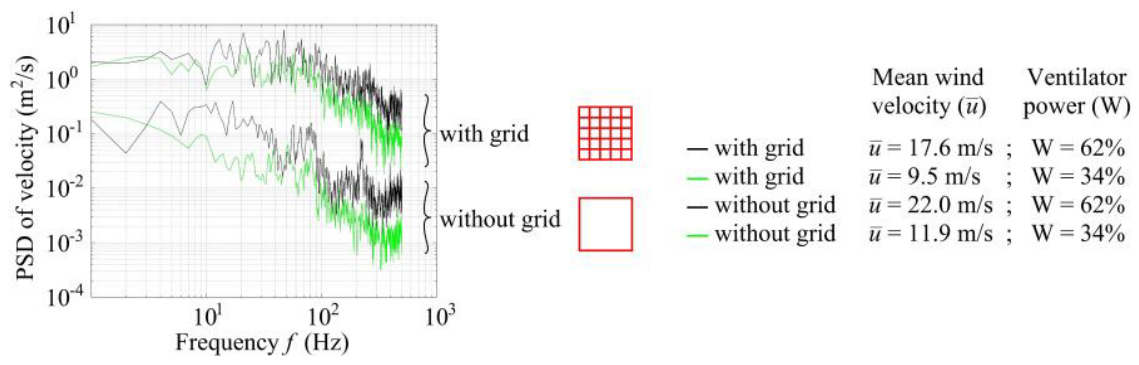

Fig. 4. PSDs of the airflow velocity in front of the model between the end-plates of the special frame measured in a smooth and moderate turbulent flow conditions. 


\section{Experimental results and discussion}

In order to verify of the adopted methodology, described in Section 3, as well as to examine the possibility of a tunnel blockage effect on the test results, the $S t$ value of a circular smooth cylinder was investigated in a calibration test with the same experimental arrangement that was used for the iced cable model. The diameter of the cylinder was 0.10 $\mathrm{m}$. The mean free stream velocity was in the range between 3.6 and $17.0 \mathrm{~m} / \mathrm{s}$. The corresponding Re range was from $2.1 \cdot 10^{4}$ to $10.2 \cdot 10^{4}$.

According to the statement given in Section 1, one should be aware that the main effect of free stream turbulence for the smooth cylinder is to develop transition to so-called the critical and transcritical regimes at a lower $R e$ values as well as to produce the greater $S t$ values than in the case of a smooth free stream. Fig. 5c shows the variation of $S t$ of the circular smooth cylinder with $R e$ for various turbulence intensity $T_{i}$ noted by Cheung and Melbourne [3] based on an experimental data.

Fig. 5a shows an example of the PSD of the airflow velocity in the wake behind the circular smooth cylinder corresponding to the mean free stream velocity of $\bar{u}=4.2 \mathrm{~m} / \mathrm{s}$. Fig. $5 \mathrm{~b}$ depicts the variation of $S t$ of the smooth cylinder with $R e$ for various $T_{i}$ based on the experimental results obtained from the calibration test at the turbulence intensity of $T_{i}=$ $12 \%$ and provided from the paper [3], i.e. from Fig. 5c, within the entire $R e$ range that was studied in this paper (the Re range was indicated in Fig. 5c by the gray field). All $S t$ values obtained in the calibration test at $T_{i}=12 \%$ were changed from 0.184 to 0.265 and were matched well with those reported in the paper [3]. Therefore, it was proved that the adopted methodology of the investigations was suitable and any corrections for a blockage effect were not needed.

a)

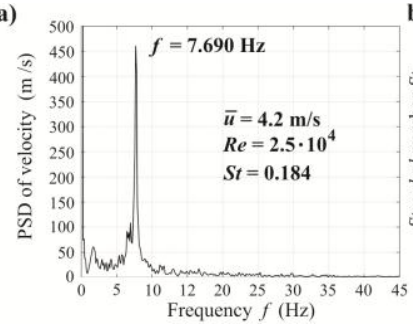

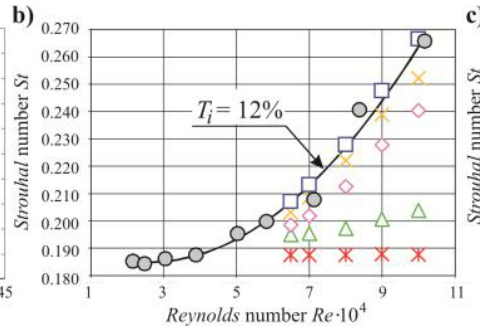

Fig. 5. (a) PSD of the airflow velocity behind the cylinder and dominant $f_{s}$ corresponding to $\bar{u}=4.2$ $\mathrm{m} / \mathrm{s}$, (b) variation of $S t$ with $R e$ in the studied range for various $T_{i}$ based on the experimental results from the calibration test at $T_{i}=12 \%$ and the paper [3], and (c) variation of $S t$ with $R e$ for various $T_{i}$ reported in the paper [3].
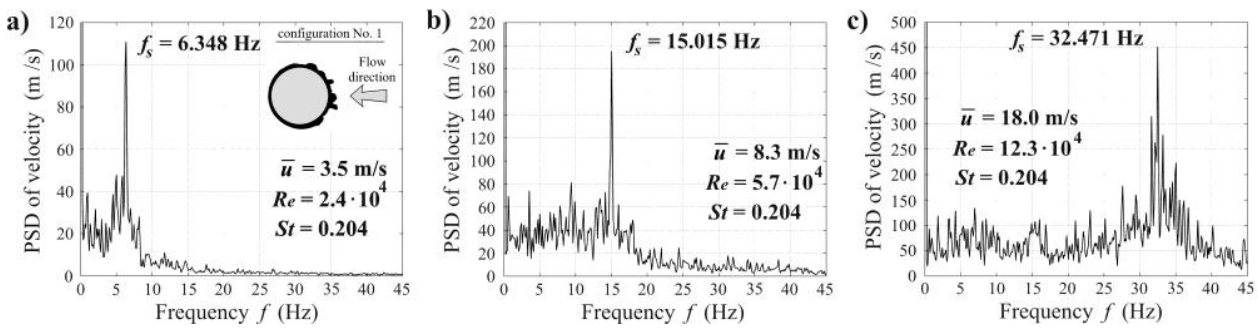

Fig. 6. Selected PSDs of the airflow velocity behind the iced cable model and dominant $f_{s}$ for configuration No. 1 corresponding to: (a) $\bar{u}=3.5 \mathrm{~m} / \mathrm{s}$, (b) $\bar{u}=8.3 \mathrm{~m} / \mathrm{s}$, and (c) $\bar{u}=18.0 \mathrm{~m} / \mathrm{s}$.

Figs 6,8 and 10 depict selected PSDs of the airflow velocity behind the iced cable model for configurations No. 1, 2 and 3, respectively, measured by CTA below the model at various $\bar{u}$ in the range of $3.5 \mathrm{~m} / \mathrm{s}$ to $18.0 \mathrm{~m} / \mathrm{s}$. Figs $7 \mathrm{a}, 9 \mathrm{a}$ and $11 \mathrm{a}$ show the variation of 
$f_{s}$ with $\bar{u}$, and Figs $7 \mathrm{~b}, 9 \mathrm{~b}$ and $11 \mathrm{~b}$ depict the variation of $S t$ with Re for configurations No. 1, 2 and 3, respectively. All St values obtained for each configuration were approximated by a continuous line. The obtained results were related to the results for the smooth cylinder at $T_{i}=12 \%$ which were indicated as a dotted line in Figs 7, 9 and 11.
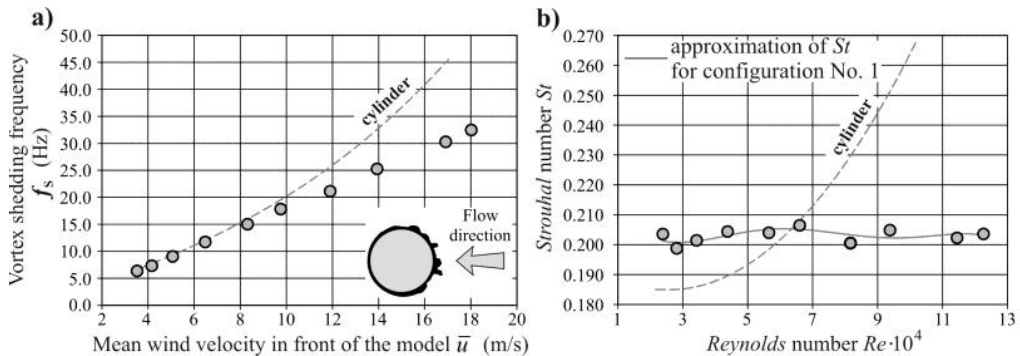

Fig. 7. (a) Variation of $f_{s}$ with $\bar{u}$, and (b) variation of $S t$ with $R e$ for configuration No. 1 with reference to the results for cylinder (dotted line).
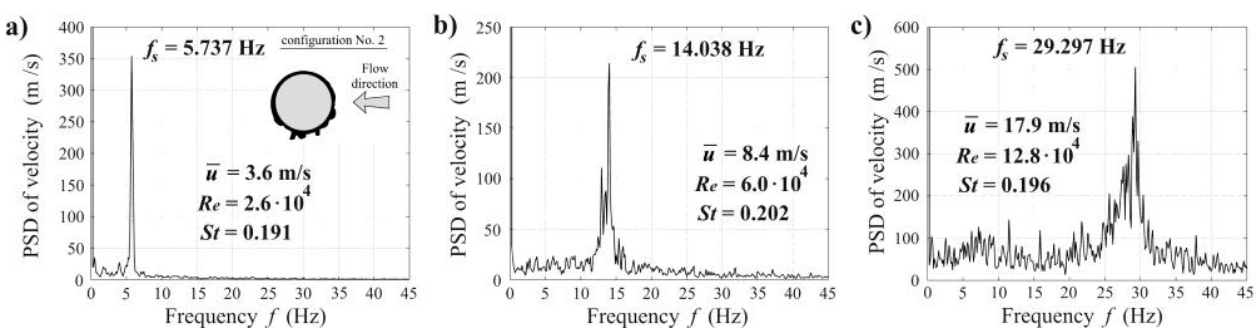

Fig. 8. Selected PSDs of the airflow velocity behind the iced cable model and dominant $f_{s}$ for configuration No. 2 corresponding to: (a) $\bar{u}=3.6 \mathrm{~m} / \mathrm{s}$, (b) $\bar{u}=8.4 \mathrm{~m} / \mathrm{s}$, and (c) $\bar{u}=17.9 \mathrm{~m} / \mathrm{s}$.
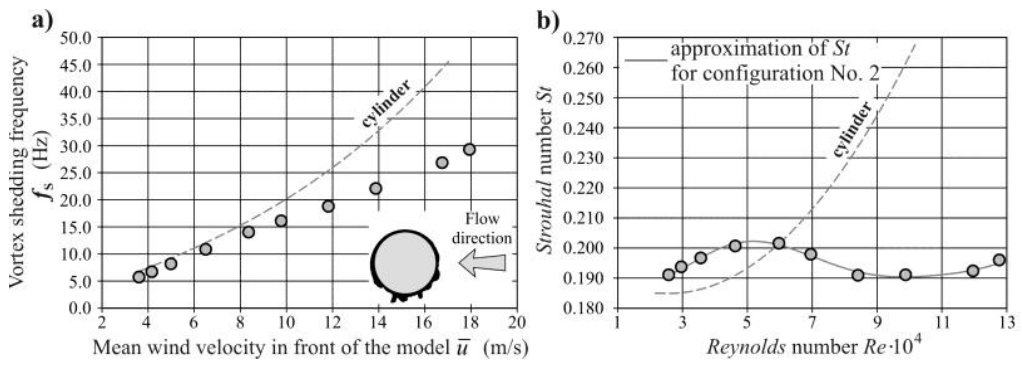

Fig. 9. (a) Variation of $f_{s}$ with $\bar{u}$, and (b) variation of $S t$ with $R e$ for configuration No. 2 with reference to the results for cylinder (dotted line).
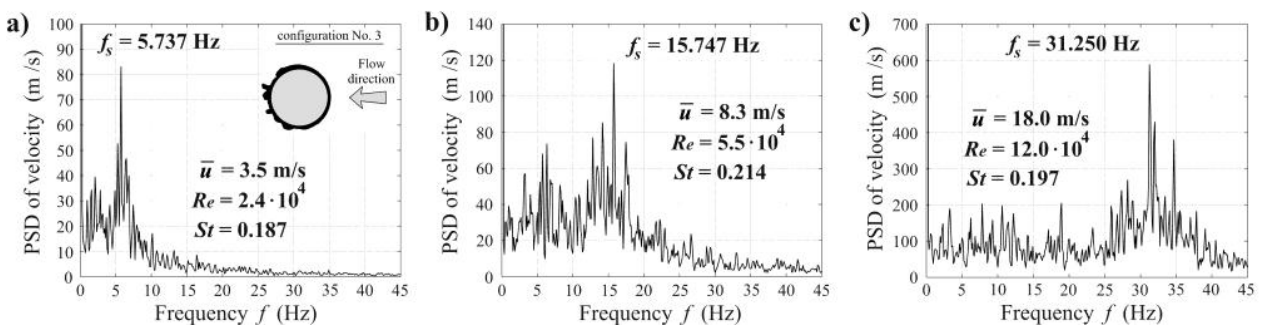

Fig. 10. Selected PSDs of the airflow velocity behind the iced cable model and dominant $f_{s}$ for configuration No. 3 corresponding to: (a) $\bar{u}=3.5 \mathrm{~m} / \mathrm{s}$, (b) $\bar{u}=8.3 \mathrm{~m} / \mathrm{s}$, and (c) $\bar{u}=18.0 \mathrm{~m} / \mathrm{s}$. 

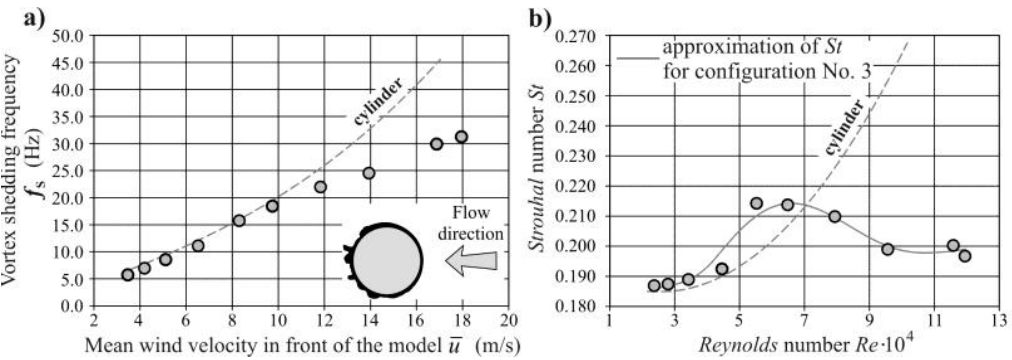

Fig. 11. (a) Variation of $f_{s}$ with $\bar{u}$, and (b) variation of $S t$ with $R e$ for configuration No. 3 with reference to the results for cylinder (dotted line).

As it was stated in the paper [9], the effect of cable inclination in the vertical plane on $S t$ in two principal practical cases should be considered in the following manner: (i) for the wind direction across the longitudinal axis of a bridge the cable inclination is unimportant and the $S t$ results obtained for configuration No. 2 are suitable, and (ii) for the wind direction along the longitudinal axis of a bridge the $S t$ results obtained for configuration No. 1 or 3 should be taken into account with the reference Reynolds number $R e_{\text {ref }}$ related to the mean wind velocity in the horizontal plane, calculated according to the formula

$$
R e_{r e f}=\frac{R e}{\sin (\alpha)}
$$

where $\alpha$ is the angle of a cable inclination in the vertical plane.

\section{Conclusion}

The experimental icing of an inclined cable model of a cable-supported bridge was carried out in the Climatic Wind Tunnel Laboratory of the Czech Academy of Sciences in Telč. The $S t$ values were examined for the stationary iced cable model with respect to three principal angles of wind attack within the range of $R e$ between $2.4 \cdot 10^{4}$ and $12.9 \cdot 10^{4}$ at the flow turbulence intensity of the order of $12 \%$.

In order to ensure the correct interpretation of the test results for the iced cable model, in the first stage, the $S t$ values was investigated for the circular smooth cylinder with the same experimental arrangement. The $S t$ results obtained at the mean turbulence intensity about $12 \%$ matched well with those reported in the literature within the Re range that was studied in this paper, i.e. from $R e=2.1 \cdot 10^{4}$ to $10.2 \cdot 10^{4}$. Therefore, the adopted methodology of the $S t$ investigations was confirmed as suitable.

St determined for configuration No. 1 has approximately a constant value, slightly varies in the range from 0.199 to 0.207 and seems to be independent of $R e$ in the range that was studied (see Fig. 7b). For $R e<6.6 \cdot 10^{4}$ the $S t$ values obtained for configuration No. 1 are greater than for the smooth cylinder. The maximum difference in this $R e$ range was about $8 \%$. For $R e>6.6 \cdot 10^{4}$ the $S t$ values for the smooth cylinder are significantly greater than for configuration No. 1 .

$S t$ determined for configuration No. 2 depends slightly on $R e$ (see Fig. 9b). Within the range of $R e=2.6 \cdot 10^{4}-5.2 \cdot 10^{4}$ the $S t$ values increase from $S t=0.191$ to 0.202 . Within the range of $R e=5.2 \cdot 10^{4}-9.9 \cdot 10^{4} S t$ decreases to minimum value of $S t=0.191$, and for $R e>$ $9.9 \cdot 10^{4} S t$ again increases to $S t=0.196$. Similarly to configuration No. 1 , also in this case for $R e>6.0 \cdot 10^{4}$ the $S t$ values obtained for configuration No. 2 are greater than for the smooth cylinder with the maximum difference of about $6 \%$. For $R e>6.0 \cdot 10^{4}$ the $S t$ values for the smooth cylinder are significantly greater than for configuration No. 2 . 
St determined for configuration No. 3 strictly depends on Re (see Fig. 11b). Within the range of $R e=2.4 \cdot 10^{4}-6.5 \cdot 10^{4} S t$ increases nonlinearly from 0.187 to 0.214 . Within the range of $R e=6.5 \cdot 10^{4}-10.1 \cdot 10^{4} S t$ decreases from $S t=0.214$ to 0.198 and for $R e>$ $10.1 \cdot 10^{4} S t$ has a nearly constant value $S t=0.199$. The $S t$ values obtained for configuration No. 3 are greater than for the smooth cylinder for $R e<7.0 \cdot 10^{4}$ with the maximum difference of about $8 \%$. For $R e>7.0 \cdot 10^{4}$ the $S t$ values for the smooth cylinder are significantly greater than for configuration No. 3 .

The differences between $f_{s}$ obtained from the measurements using CTA located above and below the model did not exceed $6 \%$ for three considered configurations of the ice cable model.

Based on the experimantal data it was shown that the ice accreated on the bridge circular cables has a significant impact on the dependence of $S t$ with $R e$ as well as on the $S t$ values within the entire $R e$ range that was studied. Therefore, it is very advisable to conduct further such studies for various ice shapes accreted on a bridge cables in another weather conditions.

This work was supported from the funds for the research and development works and connected tasks serving the development of young researchers and $\mathrm{PhD}$ students financed within the internal competition under The Minister of Science and Higher Education No. DS-MN/40/WBiA/16 and Centre of Excellence Telč sustainability project LO1219 (SaDeCET) of the Ministry of Education Youth and Sport of the Czech Republic.

\section{References}

1. M.M. Zdravkovich, Flow around circular cylinders (Oxford Sciences Publications, 2007)

2. G. Buresti, J. Wind Eng. Ind. Aerod. 8, 105-114 (1981)

3. J.C.K. Cheung, W.H. Melbourne, J. Wind Eng. Ind. Aerod. 14, 399-410 (1983)

4. Z. Savor, J. Radic, G. Hrelja, Bridge Struct. 2, 97-106 (2006)

5. H. Gjelstrup, C.T. Georgakis, A. Larsen, Wind Struct. 15, 385-407 (2012)

6. C. Demartino, H.H. Koss, C.T. Georgakis, F. Ricciardelli, J. Wind Eng. Ind. Aerod. 138, 98-119 (2015)

7. C. Demartino, F. Ricciardelli, J. Fluid Struct. 52, 81-100 (2015)

8. H. Gjelstrup, C.T. Georgakis, A. Larsen, A., Wind Struct. 15, 385-407 (2012)

9. P. Górski, S. Pospišil, S. Kuznetsov, M. Tatara, A. Marušić, Wind Struct. 22, 253-272 (2016)

10. S. Kuznetsov, S. Pospíšil, R. Král, Technical Transactions 12(2-B), 303-316 (2015)

11. S. Pospíšil, A. Buljac, H. Kozmar, S. Kuznetsov, M. Macháček, R. Král, J. Bridge Eng. (to be published)

12. A. Trush, S. Pospíśil, S. Kuznetsov, H. Kozmar, J. Bridge Eng. (to be published) 